\title{
Flexible here-and-now decisions for two-stage multi-objective optimization: method and application to energy system design selection
}

\author{
Dinah Elena Hollermann ${ }^{1}$ - Marc Goerigk ${ }^{2}$. Dörthe Franzisca Hoffrogge ${ }^{1}$. \\ Maike Hennen ${ }^{1}$. André Bardow ${ }^{1,3}$
}

Received: 20 June 2019 / Revised: 7 April 2020 / Accepted: 19 June 2020 / Published online: 13 July 2020

(c) The Author(s) 2020

\begin{abstract}
The synthesis of energy systems is a two-stage optimization problem where design decisions have to be implemented here-and-now (first stage), while for the operation of installed components, we can wait-and-see (second stage). To identify a sustainable design, we need to account for both economical and environmental criteria leading to multi-objective optimization problems. However, multi-objective optimization does not lead to one optimal design but to multiple Pareto-efficient design options in general. Thus, the decision maker usually has to decide manually which design should finally be implemented. In this paper, we propose the flexible here-and-now decision (flex-hand) approach for automatic identification of one single design for multi-objective optimization. The approach minimizes the distance of the Pareto front based on one fixed design to the Pareto front allowing multiple designs. Uncertainty regarding parameters of future operations can be easily included through a robust extension of the flex-hand approach. Results of a real-world case study show that the obtained design is highly flexible to adapt operation to the considered objective functions. Thus, the design provides an energy system with the ability to adapt to a changing focus in decision criteria, e.g., due to changing political aims.
\end{abstract}

Keywords Multi-objective optimization · Automatic solution selection · Energy system design · Two-stage optimization · Robust optimization

\section{Introduction}

The design of sustainable energy systems needs to balance multiple objectives representing economical, environmental, and social decision criteria. The resulting design

Marc Goerigk

marc.goerigk@uni-siegen.de

Extended author information available on the last page of the article 
problem is therefore best addressed by multi-objective optimization. However, multiobjective optimization does not yield an optimal design but a Pareto front with many different designs. Hence, the decision maker is often confronted with the question: How to select one single design for final implementation? To answer this question we propose the flexible here-and-now decision (flex-hand) approach.

The remaining article is structured as follows: Sect. 1 introduces the considered problem class and provides basic definitions, followed by a brief overview of existing decision support approaches including the research contribution of the article. In Sect. 2, we introduce the flex-hand approach and provide an illustrative example as well as an extension for uncertain input values. In the following Sect. 3, a real-world case study of an industrial park is introduced and the results are evaluated. We give a summary and conclusions in Sect. 4.

\subsection{Two-stage multi-objective optimization}

Two-stage optimization depends on two stages of decision making (Ben-Tal et al. 2004). Thus, there are two sets of variables: $\mathcal{X}^{f}$, the set of feasible solutions for first-stage variables $x^{f}$, and $\mathcal{X}^{s}\left(x^{f}\right)$, the set of feasible solutions for the second-stage variables $x^{s}$ which depends on the chosen first-stage solution. First-stage variables $x^{f}$ are also called here-and-now variables since once these variables are fixed, they cannot be adapted later. Second-stage variables $x^{s}$ are also called wait-and-see variables since they can still be adapted later. As an example, first-stage variables $x^{f}$ may model an investment in heating equipment (a design decision), while second-stage variables $x^{s}$ model the way the equipment is run (an operational decision).

In this paper, we use multiple objectives to design sustainable energy systems. As objectives might be conflicting, we are interested in a set of trade-off solutions. A solution is called Pareto efficient if there is no other solution that is at least as good in each objective and strictly better in at least one objective (Ehrgott 2005). For a twostage problem as energy system optimization, the two-stage multi-objective problem with $K$ objectives is given by:

$$
\begin{aligned}
& \min \left(f_{1}\left(x^{f}, x^{s}\right), \ldots, f_{K}\left(x^{f}, x^{s}\right)\right) \\
& \text { s. t. } x^{f} \in \mathcal{X}^{f} \\
& \qquad x^{s} \in \mathcal{X}^{s}\left(x^{f}\right) .
\end{aligned}
$$

In this article, we assume the set of Pareto-efficient solutions to be discrete and denote them as $\left(\chi_{1}^{f}, \chi_{1}^{s}\right), \ldots,\left(\chi_{N}^{f}, \chi_{N}^{s}\right)$. The set of Pareto-efficient solutions in the objective space, called Pareto front, is denoted as $\mathcal{P}^{*}=\left\{p_{1}, \ldots, p_{N}\right\}$ with $p_{j}=$ $\left(\rho_{1 j}, \ldots, \rho_{K j}\right)=\left(f_{1}\left(\chi_{j}^{f}, \chi_{j}^{s}\right), \ldots, f_{K}\left(\chi_{j}^{f}, \chi_{j}^{s}\right)\right) \in \mathbb{R}^{K}$. In general, not all multiobjective problems have a discrete set of Pareto-efficient solutions. In case of an infinite number of solutions, we can still apply our approach to a representative subset of Pareto-efficient solutions. A representative, discrete set of Pareto-efficient solutions can be obtained, e.g., by using the $\varepsilon$-constraint method (e.g., see Ehrgott 2005). For upper and lower bounds of the value of $\varepsilon$, the ideal and the nadir point could be chosen 
limiting the possible range of objective functions. If the ideal and the nadir point do not exist for the regarded problem, bounds have to be defined manually by the decision maker (Miettinen 2008) or an a priori approach has to be employed to restrict the regarded region of interest, e.g., by finding knee-regions of the Pareto front (see Sect. 1.2). In contrast to the points on the Pareto front, we do not assume the feasible sets $\mathcal{X}^{f}$ and $\mathcal{X}^{s}\left(x^{f}\right)$ to be discrete.

We call the problem ideal when both first-stage variables $x^{f}$ and second-stage variables $x^{s}$ can be chosen separately for each efficient solution of the problem $M \mathcal{O O}^{\mathcal{T}}$. The corresponding set of Pareto-efficient solutions in the objective space $\mathcal{P}^{*}$ is called ideal Pareto front. The word ideal emphasizes that the ideal Pareto front is always better than the Pareto front with fixed first-stage variables. In energy system optimization, the ideal Pareto front would imply changing design options (e.g. heating equipment) along the Pareto front and thus, cannot be reached by energy systems implemented in the real world.

\subsection{Decision support approaches}

In literature, several approaches have been suggested to support the decision maker in the selection of one solution from solutions on the Pareto front of multi-objective optimization problems. Some approaches reduce the number of relevant solutions, so that the decision maker has then to choose one of fewer options. The approaches can be categorized into three kinds: a priori, interactive, and a posteriori approaches (Miettinen 2008). A method to focus on the relevant solutions a priori by excluding solutions before the optimization is proposed by Branke et al. (2004) and Rachmawati and Srinivasan (2009). They introduce a preference-based evolutionary approach focusing on calculating "knee" regions of the Pareto front. Hennen et al. (2017) focus on Paretoefficient solutions which are near-optimal with respect to an aggregated criterion that represents the overall set of objective functions.

Besides a priori approaches, interactive decision making approaches exist with interaction between human and the algorithm during the solution process (Vallerio et al. 2015). Wierzbicki (2007) discuss reference point approaches where the decision maker introduces his/her preferences by updating a reference point. Employing the NIMBUS approach by Miettinen and Mäkelä (1996, 2002), the decision maker assigns the considered objectives to categories indicating which objectives should be improved and which ones could be worsened. Vallerio et al. (2015) propose an approach based on the trade-off concept. In their approach, the decision maker updates the scalarization parameters assigned to each objective. As objective the operational risks are additionally considered accounting for parametric uncertainties in the model. Eskelinen et al. (2008) introduce the Pareto navigator which helps the decision maker to explore the Pareto front in order to identify promising regions. A more detailed overview on interactive decision making approaches can be found in Miettinen (2008). Interactive decision making depends on additional input of the decision maker. Hence, an automatic selection of one solution is not possible which might be important if preferences are uncertain or changing. 
An a posteriori approach to reduce the set of relevant Pareto-efficient solutions is to cluster solutions, e.g., based on subtractive clustering (Zio and Bazzo 2011), by $k$-means classification (Taboada et al. 2007), or by a self-organizing map (Li et al. 2009). Das (1999) focus on relevant Pareto-efficient solutions by evaluating subsets of the objective functions. The approach has been further developed by Antipova et al. (2015). But still, in all approaches which reduce the number of relevant solutions, the decision maker has to select the finally implemented design.

For further reduction of the Pareto-efficient solutions, Taboada et al. (2007) and Abubaker et al. (2014) propose ranking methods which are based on a prioritization of objective functions by the decision maker. For ranking solutions without explicit prioritization, the methods LINMAP (Linear Programming Technique for Multidimensional Analysis of Preference; Srinivasan and Shocker 1973), VIKOR (Duckstein and Opricovic 1980; Opricovic and Tzeng 2004), and TOPSIS (technique for order preference by similarity to an ideal solution; Hwang and Yoon 1981; Chen and Hwang 1992) measure the distance to the ideal point and, in TOPSIS, additionally to the nadir point. The ideal point is assembled by the respective single-objective optimal values of the objective functions, the nadir point by the worst possible values. The idea of the three approaches is based on compromise programming, where the solution with objective values "as close as possible" to the ideal point is chosen, or "as far away as possible" from the nadir point (Zelany 1974). With the aim to determine key players in social networks, de la Fuente et al. (2018) employ eleven methods for automatic solution selection within the set of Pareto-efficient solutions. These include, e.g., shortest distance to the ideal point (Padhye and Deb 2011), shortest distance to all points, highest hypercube (Beume et al. 2009), and consensus (Pérez et al. 2017). Singlestage approaches for a posteriori decision making have been recently reviewed by Jing et al. (2019). For general multi-objective problems, the introduced ranking methods are suitable to select one solution. However, for two-stage problems, as the design of energy systems, the reviewed approaches could miss well-performing solutions as the approaches depend on pre-calculated efficient solutions. Thereby, well-performing designs which are not part of the pre-calculated Pareto front are not taken into account for final selection.

In particular, design optimization of energy systems is a two-stage optimization problem (Lin et al. 2016). Two-stage optimization problems consist of two sets of decision variables: The here-and-now variables representing the first stage which need to be fixed in the beginning, and the wait-and-see variables representing the second stage which can still be adapted later (Ben-Tal et al. 2004). In energy system optimization, the here-and-now variables correspond to the design of energy systems while the operation is determined by the wait-and-see variables on the second stage.

The approaches for solution reduction discussed so far do not take any advantage of the two-stage characteristics of energy systems. Thus, these approaches miss the opportunity to choose a first-stage solution which provides high flexibility on the second stage. Exploiting flexibility might be particular important, since future conditions are not known today. Hence, the capability to adapt operation to changing circumstances should be targeted (Shang and Kokossis 2005). Political aims might change; and thus, the importance of economical, environmental, and social aims might change. 
As a result, the sustainable energy system should provide flexible operation to enable an adaptation to a changing focus within the regarded criteria.

Two-stage characteristics are regarded in design selection by Mattson and Messac (2003). In their approach, the design options need to be discrete. For each design option, the corresponding Pareto front is generated separately. Afterwards, a Pareto filter is applied simultaneously to all generated fronts deleting all dominated solutions. Finally, design options lying inside a pre-defined region of interest are selected. However, with increasing number of components in the given superstructure, the design options along the Pareto front might change more frequently within the region of interest. Hence, a higher number of suitable design options needs to be taken into account during final selection. A similar approach is proposed by Carvalho et al. (2012): Based on discrete design options, the corresponding Pareto front is generated. Design options with the ability to undergo large changes in operation enable higher resilience and are thus favored. Guo et al. (2013) introduce a twostage optimal planning and design method for combined cooling, heating, and power microgrid systems. On the first stage, the system design is optimized using a generic multi-objective optimization approach. For several obtained designs, the operational costs are minimized on the second stage. Wang et al. (2019) additionally regard a feedback from the second stage to the first stage to ensure the accuracy of the planning. However, an automatic selection of favored design options is not proposed in either approaches, e.g., by applying distance measures to assess the generated Pareto fronts.

All discussed approaches do either take advantage of the two-stage characteristic of energy systems or propose a ranking of Pareto-efficient solutions. How to select one single design exploiting the two-stage nature has not been proposed so far to the authors' best knowledge.

In this paper, we propose the flex-hand approach to identify one single design which represents the whole Pareto front best without depending on any additional information of the decision maker. In our flex-hand approach, we use the ideal Pareto front as benchmark for evaluating Pareto fronts with fixed design options, i.e., with fixed first-stage variables. For this purpose, we minimize the distance between the Pareto front of the synthesis problem, i.e., the Pareto front with changing design options on the first stage, and the Pareto front induced by one fixed design. The fixed design with the minimal distance is selected by the proposed approach, since this design provides high flexibility regarding the considered objective functions. Thus, the second stage can be well adapted to a changing focus from one to another objective function.

Since not only future aims are uncertain but also future parameter values such as demands or costs, we extend our proposed approach by considering uncertain input data based on scenarios. Uncertainties of input data have been regarded, e.g., by Quintana et al. (2017), Tock and Maréchal (2015) and Lemos et al. (2018), using the sensitivity against uncertainties to assess Pareto-efficient solutions. Gabrielli et al. (2019) propose an approach in which Pareto-efficient designs are assessed by performance indicators measuring the robustness and the cost optimality. The final selection of one design depends on the target levels which need to be provided by the decision maker. Ide and Schöbel (2016) provide an overview of approaches for one-stage robust 
multi-objective problems. Robust multi-objective optimization has been applied to energy systems by Majewski et al. (2017). Sun et al. (2018) propose a multi-objective discrete robust optimization algorithm to identify a single solution by converting multiple objective functions into one unified cost function. Until now, taking uncertainties into account when automatically identifying one single design for two-stage problems has been an open research question.

For certain and uncertain two-stage problems, our approach allows automatic selection of one design regarding multiple decision criteria. While we introduce our approach in the context of energy systems, the methodology is general and can be applied to any two-stage multi-objective optimization problem.

\section{The flex-hand approach for design selection in multi-objective optimization}

The selection of energy system designs considering multiple criteria is complex, since the Pareto front can contain a diverse set of design options. To help the decision maker implementing one fixed design which allows flexible operation, we propose the flexhand approach. The approach automatically identifies the best possible design looking even beyond the set of Pareto-efficient solutions. We present the flex-hand approach in Sect. 2.1 and provide an illustrative example in Sect.2.2. In Sect.2.3, we introduce the robust extension of the flex-hand approach.

\subsection{The flexible here-and-now decision approach}

The goal of the flex-hand approach is to find one fixed design which represents best the ideal Pareto front of the two-stage multi-objective problem $\mathcal{M O O}{ }^{\mathcal{T} \mathcal{S}}$ allowing multiple design options. In optimization of energy systems, the operation of an installed system can be adapted but changing the installed system design is not possible in the short term. The flex-hand approach chooses the design with the highest ability to approximate the ideal Pareto front of the two-stage multi-objective problem $\mathcal{M O O}^{\mathcal{T}}$ by adapting operation. To determine such a highly flexible design, the flex-hand approach minimizes the distance between the ideal Pareto front and the Pareto front based on one fixed design but flexible operation. We provide an illustrative example of the flex-hand approach in Sect.2.2.

The flex-hand approach is not limited to energy system optimization but can be applied to any two-stage multi-objective problem where the first-stage variables $x^{f}$ need to be fixed right now and the second-stage variables $x^{s}$ can be determined later.

For one fixed design, i.e., for a given first-stage solution $x^{f}$, we calculate Paretoefficient solutions:

$$
\begin{aligned}
& \min \left(f_{1}\left(x^{f}, x^{s}\right), \ldots, f_{K}\left(x^{f}, x^{s}\right)\right) \\
& \text { s. t. } x^{s} \in \mathcal{X}^{s}\left(x^{f}\right) .
\end{aligned}
$$


Fig. 1 Comparison of ideal Pareto front to an arbitrary fixed first-stage Pareto front, to assess the quality of the fixed first-stage solutions $x^{f}$; dark green dots: ideal Pareto front used as benchmark; orange circles: fixed first-stage Pareto front with distance $\varepsilon$ to the ideal Pareto front; lines are included to guide reader's eyes

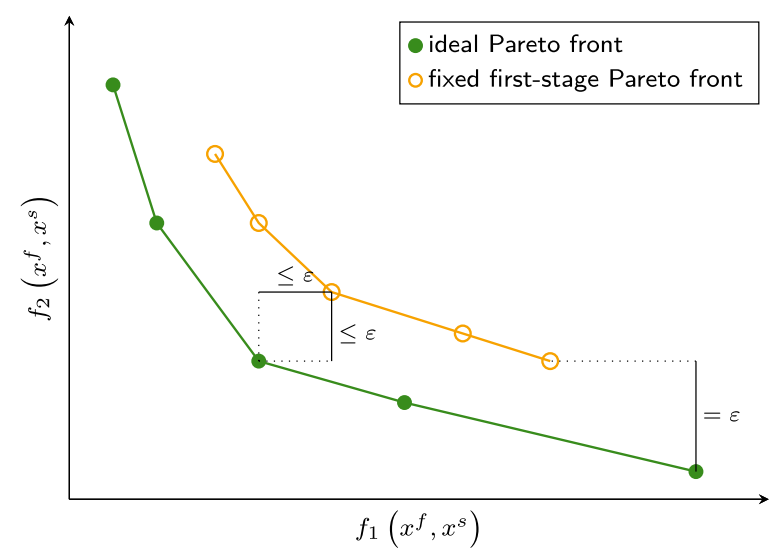

We obtain a Pareto front $\mathcal{P}\left(x^{f}\right)=\left\{p_{1}\left(x^{f}\right), \ldots, p_{N\left(x^{f}\right)}\left(x^{f}\right)\right\}$ depending on the firststage solution $x^{f}$ with $N\left(x^{f}\right)$ points which we call fixed first-stage Pareto front.

Now the question arises: How to select first-stage variables $x^{f}$ such that we get the "best" design? Each point on the ideal Pareto front is part of at least one fixed firststage Pareto front. However, the flex-hand approach looks even beyond the solutions on the ideal Pareto front. To determine the quality of an arbitrary first-stage solution $x^{f}$, we compare the Pareto front $\mathcal{P}\left(x^{f}\right)$ with fixed first-stage to the ideal Pareto front $\mathcal{P}^{*}$. For the comparison of two sets of multi-objective solutions, a variety of distance measures have been developed (for a review see Zitzler et al. 2003). Here, we choose a comparison metric based on an additive binary $\varepsilon$-indicator. As discussed by Zitzler et al. (2003), there is no single best way to compare Pareto fronts, but the $\varepsilon$-indicator is proposed as a good overall method. Employing other metrics would be possible in our setting.

For two sets $\mathcal{P}^{1}=\left\{p_{1}, \ldots, p_{S}\right\}$ and $\mathcal{P}^{2}=\left\{q_{1}, \ldots, q_{T}\right\}$ in the $K$-dimensional objective space, the binary $\varepsilon$-indicator is obtained by

$$
I\left(\mathcal{P}^{1}, \mathcal{P}^{2}\right)=\min \left\{\varepsilon: \forall l \in[T] \exists j \in[S] \text { s. t. } p_{i j}-q_{i l} \leq \varepsilon \forall i \in[K]\right\}
$$

where we use the notation $[Z]=\{1, \ldots, Z\}$ for sets with any integer $Z \in \mathbb{N}$. For our approach, the measure $I\left(\mathcal{P}\left(x^{f}\right), \mathcal{P}^{*}\right)$ indicates the distance between a fixed first-stage Pareto front and the ideal Pareto front.

The comparison metric can be interpreted as follows: Recall that each point of the ideal Pareto front $\mathcal{P}^{*}$ may involve changing first-stage solutions $x^{f}$ along the front. In contrast, the fixed first-stage Pareto front $\mathcal{P}\left(x^{f}\right)$ is based on one single first-stage solution $x^{f}$ where only the second-stage decision can be adapted. Figure 1 shows the comparison of the ideal Pareto front to an arbitrary fixed first-stage Pareto front.

For each point on the ideal Pareto front, a point on the fixed first-stage Pareto front can be determined such that the difference in each objective function is smaller than a value of $\varepsilon$. By minimizing $\varepsilon$, the distance between the Pareto fronts is minimized. In this way, for energy systems, we minimize the distance between the ideal Pareto 
front allowing changing designs as well as operation and the Pareto front based on one fixed design where only the operation can be adapted.

Since we consider the difference regarding each objective function separately, we normalize the objective functions based on the value range in $\mathcal{P}^{*}$ to circumvent misleading effects by different scales of objective values. Towards this end, we use the normalized objectives $\bar{f}_{i}$ with

$$
\bar{f}_{i}\left(x^{f}, x^{s}\right)=\frac{f_{i}\left(x^{f}, x^{s}\right)-\min _{j \in[N]} f_{i}\left(\chi_{j}^{f}, \chi_{j}^{s}\right)}{\max _{j \in[N]} f_{i}\left(\chi_{j}^{f}, \chi_{j}^{s}\right)-\min _{j \in[N]} f_{i}\left(\chi_{j}^{f}, \chi_{j}^{s}\right)} .
$$

In the following, we write $\overline{\mathcal{P}}\left(x^{f}\right)$ and $\overline{\mathcal{P}}^{*}$ to denote normalized Pareto fronts.

Searching for an optimal first-stage variable $\left(x^{f}\right)^{*}$, i.e., an optimal design, we now minimize the distance between the Pareto fronts:

$$
\min \left\{I\left(\overline{\mathcal{P}}\left(x^{f}\right), \overline{\mathcal{P}}^{*}\right): x^{f} \in \mathcal{X}^{f}\right\} .
$$

The problem formulation can also be written as:

$\min \bar{\varepsilon}$

$$
\begin{aligned}
& \text { s. t. } \bar{f}_{i}\left(x^{f}, x_{j}^{s}\right)-\bar{f}_{i}\left(\chi_{j}^{f}, \chi_{j}^{s}\right) \leq \bar{\varepsilon} \quad \forall i \in[K], j \in[N] \\
& x^{f} \in \mathcal{X}^{f} \\
& x_{j}^{s} \in \mathcal{X}^{s}\left(x^{f}\right) \quad \forall j \in[N] .
\end{aligned}
$$

Here, $\bar{f}_{i}\left(\chi_{j}^{f}, \chi_{j}^{s}\right)$ represents the normalized objective function value of point $j$ on the ideal Pareto front $\overline{\mathcal{P}}^{*}$. Note that given a first-stage solution $x^{f}$, the number of points $N\left(x^{f}\right)$ of the resulting fixed first-stage Pareto front $\overline{\mathcal{P}}\left(x^{f}\right)$ might be different from the number $N$ of points of the ideal Pareto front $\overline{\mathcal{P}}^{*}$. However, for the purpose of identifying an optimal first-stage solution $\left(x^{f}\right)^{*}$, it is sufficient to consider exactly $N$ points on the fixed first-stage Pareto front $\overline{\mathcal{P}}\left(x^{f}\right)$. The key is here that we are looking for the maximal minimum distance to the $N$ points on the ideal Pareto front $\overline{\mathcal{P}}^{*}$. Thus, it is sufficient to identify, for each point on the ideal Pareto front $\overline{\mathcal{P}}^{*}$, one point on the fixed first-stage Pareto front $\overline{\mathcal{P}}\left(x^{f}\right)$ which minimizes the distance. As a result, we need the same number of points in general, $N\left(x^{f}\right)=N$. Any additional points on the fixed first-stage Pareto front $\overline{\mathcal{P}}\left(x^{f}\right)$ (i.e., $N\left(x^{f}\right)>N$ ) would be redundant and not be chosen by the binary $\varepsilon$-indicator [Eq. (1)].

The flex-hand approach yields an optimal first-stage solution $\left(x^{f}\right)^{*}$ which represents the ideal Pareto front best regarding the chosen measure. We call the optimal first-stage solution $\left(x^{f}\right)^{*}$ the flex-hand solution. In general, the flex-hand solution chosen by our approach is not necessarily part of the solutions of the calculated ideal Pareto front $\mathcal{P}^{*}$. Thus, approaches based on sorting or solution-reduction of the ideal Pareto front would not be able to identify the flex-hand solution in general.

Having found a flex-hand solution, we calculate the corresponding Pareto-efficient second-stage solution $x^{s}$ in a separate post-processing optimization step. The resulting 
Fig. 2 Application of the flex-hand approach to a single-stage optimization problem; as there is no second stage, only one point is assessed; $\varepsilon_{1}, \varepsilon_{2}, \varepsilon_{3}$, and $\varepsilon_{4}$ represent the differences of objective values with $\varepsilon_{1}>\varepsilon_{2}$ and $\varepsilon_{3}>\varepsilon_{4}$; lines are included to guide reader's eyes

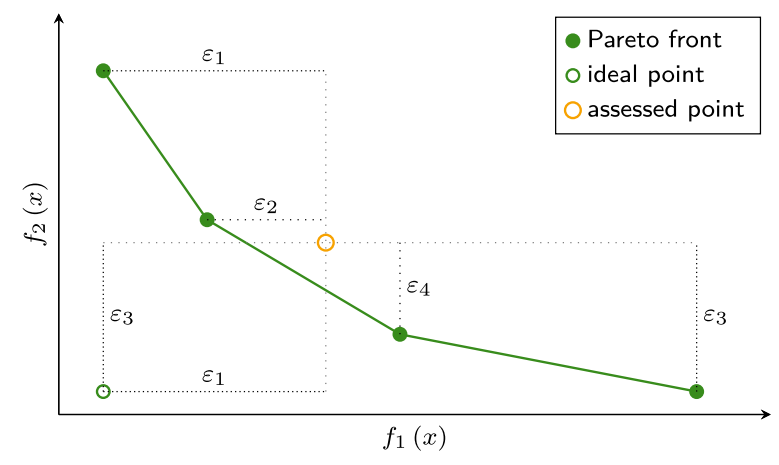

Pareto front is called the flex-hand Pareto front. The number of points on the flex-hand Pareto front might differ from the original number of points $N$. For energy systems, this post-processing optimization corresponds to an operational multi-objective optimization based on a fixed design.

Applying the flex-hand approach to single-stage optimization problems is also possible. For single-stage problems, the flex-hand approach reduces to an approach minimizing the objective-wise distance to the ideal point. As there is no second stage, the distance is measured only from a point instead of a whole Pareto front to the ideal Pareto front. Hence, only the anchor points are decisive when determining the minimal distance. As we measure the distance objective-wise, the distance is equivalent to the distance to the ideal point (Fig. 2).

As there is no second stage, the fixed first-stage Pareto front degenerates to only one point. The inner points on the Pareto front always lead to smaller differences of the objective values than one of the anchor points (in Fig. 2: $\varepsilon_{1}>\varepsilon_{2}$ and $\varepsilon_{3}>\varepsilon_{4}$ ). Thus, only the anchor points are decisive. Since the differences of the anchor points to the assessed point are identical to the differences regarding the ideal point, the flex-hand approach reduces to an approach minimizing the objective-wise distance to the ideal point.

In contrast to single-stage problems, the anchor points are not decisive in general for two-stage problems, as the following example shows.

\subsection{An illustrative example}

We now describe the flex-hand approach using a small example problem. In the example, the flex-hand approach is applied on the two-stage selection problem (see Kasperski and Zieliński 2017). In the basic problem, we need to select $p$ out of $n$ items; that is, given a cost vector $c=\left(c_{1}, \ldots, c_{n}\right)$, the (trivial) one-stage, one-criterion problem is to solve:

$$
\begin{aligned}
& \min \sum_{i \in[n]} c_{i} x_{i} \\
& \text { s. t. } \sum_{i \in[n]} x_{i}=p
\end{aligned}
$$




$$
x_{i} \in\{0,1\} \quad \forall i \in[n] .
$$

In the two-stage problem, we can decide to buy some items now, and complete these items to a full selection of $p$ items later. Hence, the sets of first-stage and second-stage feasible solutions $\mathcal{X}^{f}$ and $\mathcal{X}^{s}\left(x^{f}\right)$, respectively, are defined by:

$$
\begin{aligned}
\mathcal{X}^{f} & =\left\{x \in\{0,1\}^{n}: \sum_{i \in[n]} x_{i} \leq p\right\} \quad \text { and } \\
\mathcal{X}^{s}\left(x^{f}\right) & =\left\{x \in\{0,1\}^{n}: \sum_{i \in[n]} x_{i}+x_{i}^{f}=p, x_{i}+x_{i}^{f} \leq 1 \forall i \in[n]\right\} .
\end{aligned}
$$

Given a cost vector $C=\left(C_{1}, \ldots, C_{n}\right)$ of first-stage costs and $c=\left(c_{1}, \ldots, c_{n}\right)$ of the second-stage costs, the two-stage problem becomes:

$$
\begin{aligned}
& \min \sum_{i \in[n]} C_{i} x_{i}^{f}+c_{i} x_{i}^{s} \\
& \text { s. t. } \sum_{i \in[n]} x_{i}^{f}+x_{i}^{s}=p \\
& x_{i}^{f}+x_{i}^{s} \leq 1 \quad \forall i \in[n] . \\
& x_{i}^{f} \in\{0,1\} \quad \forall i \in[n] . \\
& x_{i}^{s} \in\{0,1\} \quad \forall i \in[n] .
\end{aligned}
$$

Now, let us assume that two objective functions are given

$$
\begin{aligned}
& f_{1}\left(x^{f}, x^{s}\right)=\sum_{i \in[n]} C_{i} x^{f}+c_{i} x_{i}^{s} \\
& f_{2}\left(x^{f}, x^{s}\right)=\sum_{i \in[n]} C_{i} x^{f}+d_{i} x_{i}^{s}
\end{aligned}
$$

with an additional cost vector $d=\left(d_{1}, \ldots, d_{n}\right)$ of second-stage costs. The ideal Pareto front is the same as the Pareto front of the problem:

$$
\begin{aligned}
& \min \left(f_{1}\left(x^{f}, x^{s}\right), f_{2}\left(x^{f}, x^{s}\right)\right) \\
& \text { s. t. } \sum_{i \in[n]} x_{i}^{f}+x_{i}^{s}=p \\
& x_{i}^{f}+x_{i}^{s} \leq 1 \quad \forall i \in[n] . \\
& x_{i}^{f} \in\{0,1\} \quad \forall i \in[n] . \\
& x_{i}^{s} \in\{0,1\} \quad \forall i \in[n] .
\end{aligned}
$$


Table 1 Cost vectors for $n=6$ items of the illustrative example

\begin{tabular}{llllrrr}
\hline item & 1 & 2 & 3 & \multicolumn{1}{c}{4} & 5 & 6 \\
\hline$C$ & 58 & 84 & 38 & 35 & 22 & 82 \\
$c$ & 60 & 48 & 17 & 100 & 3 & 27 \\
$d$ & 75 & 11 & 67 & 27 & 45 & 88 \\
\hline
\end{tabular}

Table 2 Solutions and objective function values of the ideal Pareto front of the illustrative example

\begin{tabular}{lllll}
\hline Number & $x_{i}^{f}$ & $x_{i}^{s}$ & $f_{1}$ & $f_{2}$ \\
\hline 1 & \{\} & $\{3,5,6\}$ & 47 & 200 \\
2 & $\{4\}$ & $\{3,5\}$ & 55 & 147 \\
3 & \{\} & $\{2,3,5\}$ & 68 & 123 \\
4 & $\{3,4\}$ & $\{5\}$ & 76 & 118 \\
5 & $\{4\}$ & $\{2,5\}$ & 86 & 91 \\
6 & $\{4,5\}$ & $\{2\}$ & 105 & 68 \\
7 & $\{5\}$ & $\{2,4\}$ & 170 & 60 \\
\hline
\end{tabular}

In the flex-hand approach, we take into account that it is usually not possible to change the first-stage solution $x^{f}$, even if the preference of the decision maker changes whether criterion $f_{1}$ or $f_{2}$ is more important. Hence, a first-stage solution $x^{f}$ is desired leading to a good approximation of the whole ideal Pareto front such that the decision maker obtains a satisfying solution even though his/her preference might change.

In this example, the task is to choose $p=3$ items out of $n=6$ items over the first and second stage, minimizing objective functions (2) and (3). The corresponding cost vectors providing the costs of the items are given in Table 1.

For selecting $p=3$ elements, the ideal Pareto front of problem $\mathcal{M O O} \mathcal{O}_{\mathcal{E} \mathcal{X}}^{\mathcal{I}}$ comprises seven points, see Table 2 . Note that we allow the first-stage variables $x^{f}$ to differ between solutions of the ideal Pareto front. In the following, we write $\{i\}$ if item $i$ is selected and $x_{i}$ is equal to 1 .

In the example, the flex-hand approach chooses one first-stage solution $x^{f}$ and extends it to up to seven full solutions with 3 items each such that the ideal Pareto front is approximated. Here, we dispense with the normalization in order to retain comprehensibility of the example. Table 3 presents details of an optimal flex-hand solution. Note that none of the solutions found this way are actually on the ideal Pareto front in this case and thus the selected highly flexible first-stage solution with selected item 3 would not have been identified by a posteriori selection methods.

In Table 3 we also indicate the differences in the two objective values $f_{1}$ and $f_{2}$, i.e., the objective-wise distances $\varepsilon_{1}$ and $\varepsilon_{2}$. The maximum distance $\varepsilon^{*}$ is reached in solution number six, with a difference of 26 in the second objective with costs of 94 instead of 68. Hence, the objective value of the flex-hand approach is $\varepsilon^{*}=26$. Both Pareto fronts are visualized in Fig. 3. 
Table 3 Flex-hand solution with corresponding objective function values and objective-wise distances of the illustrative example

Fig. 3 Ideal and flex-hand Pareto fronts of the illustrative example

\begin{tabular}{lllrrrr}
\hline number & $\left(x_{i}^{f}\right)^{*}$ & $x_{i}^{s}$ & $f_{1}$ & $f_{2}$ & $\varepsilon_{1}$ & $\varepsilon_{2}$ \\
\hline 1 & $\{3\}$ & $\{5,6\}$ & 68 & 171 & 21 & 0 \\
2 & $\{3\}$ & $\{5,6\}$ & 68 & 171 & 13 & 24 \\
3 & $\{3\}$ & $\{2,5\}$ & 89 & 94 & 21 & 0 \\
4 & $\{3\}$ & $\{2,5\}$ & 89 & 94 & 13 & 0 \\
5 & $\{3\}$ & $\{2,5\}$ & 89 & 94 & 3 & 3 \\
6 & $\{3\}$ & $\{2,5\}$ & 89 & 94 & 0 & 26 \\
7 & $\{3\}$ & $\{2,4\}$ & 186 & 76 & 16 & 16 \\
\hline
\end{tabular}

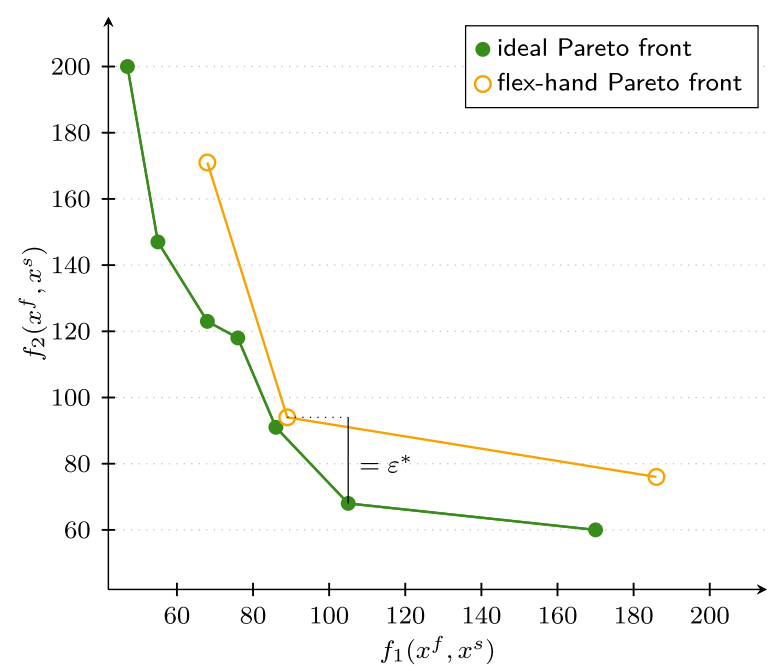

\subsection{The robust flex-hand approach}

In optimization of energy systems, decisions are based on input parameters which are inherently uncertain. Thus, we extend the proposed flex-hand approach for problems comprising uncertain parameters in the objective functions and constraints, and introduce the robust flex-hand approach. As the flex-hand approach, the robust flex-hand approach can also be applied to any other two-stage multi-objective problem where the first-stage needs to be determined in advance.

The robust flex-hand approach automatically selects first-stage solutions taking uncertainties into account. For this purpose, we assume multiple scenarios which are contained in the discrete uncertainty set $\mathcal{U}$. In each scenario, we compare the ideal Pareto front for the current scenario to a fixed first-stage Pareto front which is based on one fixed set of first-stage variables for all scenarios simultaneously. We then minimize the distance between the Pareto fronts in the worst case and thereby find the robust optimal first-stage solution.

For this purpose, we calculate the ideal Pareto front $\mathcal{P}^{*}(\xi)$ in each scenario $\xi \in \mathcal{U}$ separately. Here, the number of elements in $\mathcal{P}^{*}(\xi)$ is denoted by $N(\xi)$. The objectives 


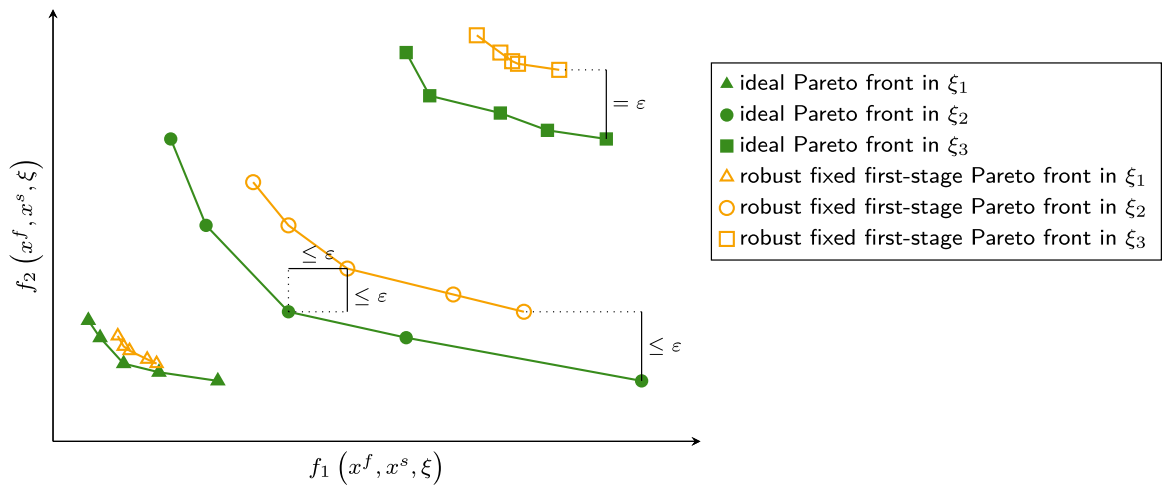

Fig. 4 Idea of the robust flex-hand approach: For each scenario separately, the ideal Pareto front (dark green filled marks) and a robust fixed first-stage Pareto front (orange unfilled marks) are compared; triangles, circles, and squares represent scenario $\xi_{1}, \xi_{2}$, and $\xi_{3}$, respectively; the distance $\varepsilon$ is calculated regarding all scenarios; here, Pareto fronts are presented without normalization; lines are included to guide reader's eyes

are parametrized also through scenarios $\xi \in \mathcal{U}$, i.e., we use $f_{i}\left(x^{f}, x^{s}(\xi), \xi\right)$. Again, we normalize objectives which we denote by $\bar{f}_{i}\left(x^{f}, x^{s}(\xi), \xi\right)$, for each scenario $\xi$. To minimize the worst-case distance between the ideal Pareto front and the fixed firststage Pareto front, we minimize the maximum value of the $\varepsilon$-indicator [Eq. (1)] over all $\xi \in \mathcal{U}$ :

$\min \bar{\varepsilon}$

$$
\begin{array}{lr}
\text { s. t. } \bar{f}_{i}\left(x^{f}, x_{j}^{s}(\xi), \xi\right)-\bar{f}_{i}\left(\chi_{j}^{f}(\xi), \chi_{j}^{s}(\xi), \xi\right) \leq \bar{\varepsilon} & \forall \xi \in \mathcal{U}, i \in[K], j \in[N(\xi)] \\
x^{f} \in \mathcal{X}^{f} & \forall \xi \in \mathcal{U}, j \in[N(\xi)] . \\
x_{j}^{s}(\xi) \in \mathcal{X}^{s}\left(x^{f}, \xi\right) & \forall \xi
\end{array}
$$

Here, $\mathcal{X}^{s}\left(x^{f}, \xi\right)$ is the set of feasible second-stage variables given a first-stage solution $x^{f}$ and a scenario $\xi$. The optimal first-stage solution $\left(x^{f}\right)^{*}$ is the identified robust flexhand solution.

An example is given in Fig. 4. The ideal Pareto front is calculated for each of the three scenarios $\left(\xi_{1}, \xi_{2}, \xi_{3}\right)$ separately. The robust fixed first-stage Pareto fronts are based on one fixed set of first-stage variables for all scenarios; however, the corresponding robust fixed first-stage Pareto fronts are calculated for each scenario separately by adapting the second-stage variables $x^{s}$.

We now compare the robust flex-hand approach with other existing robust optimization approaches (see Aissi et al. 2009; Gabrel et al. 2014; Yanıkoğlu et al. 2019 for general surveys). We can rewrite the flex-hand approach as the following problem:

$$
\min _{x^{f} \in \mathcal{X}^{f}} \max _{\xi \in \mathcal{U}} \max _{\substack{y^{f}(\xi) \in \mathcal{X}^{f}, y^{s}(\xi) \in \mathcal{X}^{s}\left(y^{f}(\xi), \xi\right)}} \min _{x^{s}(\xi) \in \mathcal{X}^{s}\left(x^{f}, \xi\right)} \max _{i \in[K]}\left(\bar{f}_{i}\left(x^{f}, x^{s}(\xi), \xi\right)-\bar{f}_{i}\left(y^{f}(\xi), y^{s}(\xi), \xi\right)\right),
$$


that is, we first decide on a first-stage solution $x^{f}$, then an adversary determines both a comparator solution $\left(y^{f}(\xi), y^{s}(\xi)\right)$ as well as a scenario $\xi \in \mathcal{U}$. We then extend $x^{f}$ with a second-stage solution $x^{s}(\xi)$ to get as close as possible to the comparator $\bar{f}_{i}\left(y^{f}(\xi), y^{s}(\xi), \xi\right)$ under scenario $\xi$. The objective value of the flex-hand approach is then determined by the index $i \in[K]$ leading to the largest difference between the achieved objective value $\bar{f}_{i}\left(x^{f}, x^{s}(\xi), \xi\right)$ and the comparator $\bar{f}_{i}\left(y^{f}(\xi), y^{s}(\xi), \xi\right)$. Because of the min-max-min-max objective, we are not in the classical settings of strict (min-max; Soyster 1973) or adjustable robustness (min-max-min; Ben-Tal et al. 2004).

Now, we consider the case of a single objective function, i.e., $K=1$. Then our flexhand approach is simplified by removing the last maximum. The resulting problem has the form of a two-stage min-max regret problem. Problems of this type are challenging to analyze and the object of current research, see Poursoltani and Delage (2019) for some theoretical insights. In particular, the optimal solution of the resulting problem might change if the uncertainty set $\mathcal{U}$ is replaced by its extreme points.

\section{Case study}

In this section, we apply the flex-hand approach to design the energy system of a real-world industrial park. The case study is introduced in Sect. 3.1. The results of the flex-hand approach are presented and discussed in detail in Sect.3.2 and in Sect.3.3 for the robust flex-hand approach.

To compute Pareto fronts, we use the adaptive normal boundary intersection method (Das and Dennis 1998). For calculation, we employ 4 threads of a computer with 3.24 $\mathrm{GHz}$ and 64 GB RAM. The problem is formulated in GAMS 24.7.3 (McCarl and Rosenthal 2016) and solved by the solver CPLEX 12.6.3.0 (IBM Corporation 2015) to machine accuracy.

\subsection{The real-world example}

The real-world example is based on our previous work (Voll et al. 2013) on the optimization of a distributed energy supply system. We consider an industrial site with one power grid, one heating grid and two separate cooling grids (Site A and Site B). The thermal demands and their uncertainties are given in Fig. 5.

The design of the energy system corresponds to sizing and installing any number of components from the following types of energy conversion components: boilers $B$, combined heat and power engines $C H P$, absorption chillers $A C$, and compression chillers $C C$. Natural gas can be used at costs of $p^{g a s}=6 \mathrm{ct} / \mathrm{kWh}$ with $\pm 40 \%$ of uncertainty. Furthermore, we assume a connection to the electricity grid. Electricity can be purchased for $p^{e l, \text { buy }}=16 \mathrm{ct} / \mathrm{kWh}$ and sold for $p^{e l, \text { sell }}=10 \mathrm{ct} / \mathrm{kWh}$. For purchasing and selling, an uncertainty of $\pm 46 \%$ is considered. All possibly uncertain input parameters depend on the scenario $\xi$ and are additionally marked using a tilde. The values for uncertainty are deduced from Majewski et al. (2017). To design a sustainable energy system, we employ an economical and an environmental objective 
Fig. 5 Thermal demands of the industrial site and their uncertainties represented by error bars; adopted from Majewski et al. (2017)

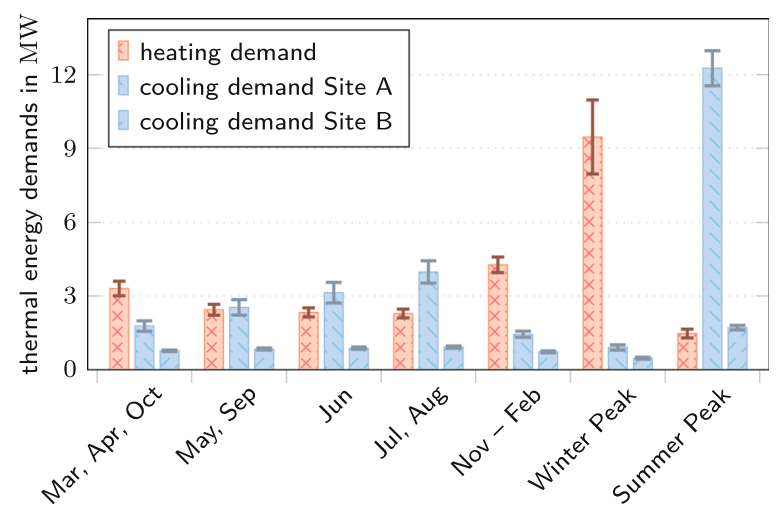

function: the total annualized costs $T A C$ and the global warming impact $G W I$. In principle, the method could also consider social criteria (Mota et al. 2015).

The total annualized costs $T A C$ are defined by:

$$
\begin{aligned}
\text { TAC } & \left(\dot{U}, \dot{U}^{\text {el,buy }}, \dot{V}^{e l, s e l l}, \operatorname{INVEST}_{k} ; \xi\right) \\
= & \sum_{t \in[T]}\left[\Delta \tau _ { t } \left(\widetilde{p}^{\text {gas }}(\xi) \cdot \sum_{k \in B \cup C H P} \dot{U}_{k t}(\xi)+\widetilde{p}^{\text {el,buy }}(\xi) \cdot \dot{U}_{t}^{e l, b u y}(\xi)\right.\right. \\
& \left.\left.-\widetilde{p}^{e l, s e l l}(\xi) \cdot \dot{V}_{t}^{e l, s e l l}(\xi)\right)\right] \\
& +\sum_{k \in \mathcal{K}}\left(\frac{1}{P V F}+p_{k}^{m}\right) \cdot \operatorname{INVEST}_{k}
\end{aligned}
$$

Here, $k$ represents a component in the set of all components $\mathcal{K}=B \cup C H P \cup A C \cup C C$ which might be installed. For each time step $t \in[T], \Delta \tau_{t}$ represents its length. The corresponding input energy flows of natural gas for boilers $B$ and combined heat and power engines $C H P$ are denoted by $\dot{U}_{k t}$. The input and the output energy flow of electricity are denoted by $\dot{U}_{t}^{e l, b u y}$ and $\dot{V}_{t}^{e l, s e l l}$, respectively. For each component $k, p_{k}^{m}$ represents the annual maintenance costs as share of the investment costs $I N V E S T_{k}$. For annualizing the investment costs INVEST ${ }_{k}$, we use the present value factor (Broverman 2010)

$$
P V F=\frac{(i+1)^{h}-1}{(i+1)^{h} \cdot i}
$$

with an interest rate $i=8 \%$ and a time horizon $h=4 \mathrm{a}$.

In the case study, the second-stage variables $x^{s}$ comprise time-dependent operational variables $\dot{U}_{k t}, \dot{U}_{t}^{\text {el, buy }}$ and $\dot{V}_{t}^{\text {el,sell }}$. The first stage consists of the design variables (see Sect. 1), besides the corresponding investment costs INVEST ${ }_{k}$. 
The global warming impact is given by:

$$
\begin{aligned}
G W I & \left(\dot{U}, \dot{U}^{\text {el, buy }}, \dot{V}^{\text {el,sell }} ; \xi\right) \\
& =\sum_{t \in[T]} \Delta \tau_{t}\left[\sum_{k \in B \cup C H P} \dot{U}_{k t}(\xi) \cdot G W I^{\text {gas }}+\left(\dot{U}_{t}^{\text {el, buy }}(\xi)-\dot{V}_{t}^{\text {el,sell }}(\xi)\right) \cdot \widetilde{G W I}^{e l}(\xi)\right] .
\end{aligned}
$$

We employ $G W I^{\text {gas }}=244 \mathrm{~g}_{\mathrm{CO}_{2} \text {-eq. }} / \mathrm{kWh}$ for the specific global warming impact of gas which is not subject to remarkable variation. For the specific global warming impact $G W I^{e l}$ of electricity purchased from the grid, we employ a value of $561 \mathrm{~g}_{\mathrm{CO}_{2} \text {-eq. }} / \mathrm{kWh}$. Since the future electricity mix might change significantly, we assume the specific global warming impact $G W I^{e l}$ to be uncertain lying within $430 \mathrm{~g}_{\mathrm{CO}_{2} \text {-eq. }} / \mathrm{kWh}$ and $610 \mathrm{~g}_{\mathrm{CO}_{2} \text {-eq. }} / \mathrm{kWh}$. When selling electricity to the grid, a credit for global warming impact is given, following the idea of the avoided burden (Baumann and Tillman 2004). Here, the global warming impact GWI depends only implicitly on the firststage variables $x^{f}$ due to the constraints. A direct influence would be given if the global warming impact induced by the manufacturing of the components was taken into account. However, since the global warming impact of the operation has usually a significantly higher impact (Guillén-Gosálbez 2011), we neglect this dependency. The complete flex-hand optimization model is presented in "Appendix A".

For the design optimization, we assume a "green field" without existing energy components. However, the flex-hand approach could also be applied to retrofit an energy system.

\subsection{The flex-hand design}

We now employ the flex-hand approach to design the sustainable energy system in order to obtain the best solution for the first-stage variables $x^{f}$ which determine the flex-hand design. For this purpose, we first calculate the ideal Pareto front as a benchmark. The ideal Pareto front is obtained by allowing a different design for each point on the front. The largest optimization problem for calculating a point on the ideal Pareto front consists of 1950 equations, 576 variables, and 310 binary variables after presolve. In total, the whole ideal Pareto front is calculated in $317 \mathrm{~s}$. The flex-hand problem has 5099 equations, 2023 variables, and 751 binary variables after presolve. Here, computing the flex-hand Pareto front takes $152 \mathrm{~s}$. Both the ideal Pareto front and the flex-hand Pareto front are shown in Fig. 6.

Compared to the fixed first-stage Pareto fronts of the ideal designs, the flex-hand Pareto front of the selected design is "stretched out". Thus, the flex-hand design allows for flexible operation providing a high ability to adapt to changing future objectives. The flex-hand design can be operated such that the total annualized costs TAC are very low at 7.8 Mio.€/a or the global warming impact $G W I$ is very low at $22.6 \mathrm{kt}_{\mathrm{CO}_{2} \text {-eq. }} / \mathrm{a}$.

In this case study, the minimal distance $\varepsilon^{*}$ between the ideal and the flex-hand Pareto front is limited, e.g., by the anchor points with minimal total annualized costs limits (Fig. 6). The corresponding scaled value for the minimized distance is $\bar{\varepsilon}^{*}=$ 0.128 . For unscaled values, the minimal total annualized costs for the ideal design are $T A C^{i d e a l}=7.51$ Mio. $€ / \mathrm{a}$ and for the flex-hand design $T A C^{\text {flex-hand }}=7.78 \mathrm{Mio} . € / \mathrm{a}$, 
Fig. 6 Comparison of ideal Pareto front (dark green dots) and the flex-hand Pareto front (orange circles) with minimal distance $\varepsilon^{*}$ to the ideal Pareto front; small dark green dots: fixed first-stage Pareto front of ideal designs, i.e.,

Pareto-efficient operation for each design of the ideal Pareto front; here, Pareto fronts are presented without normalization; lines are included to guide reader's eyes

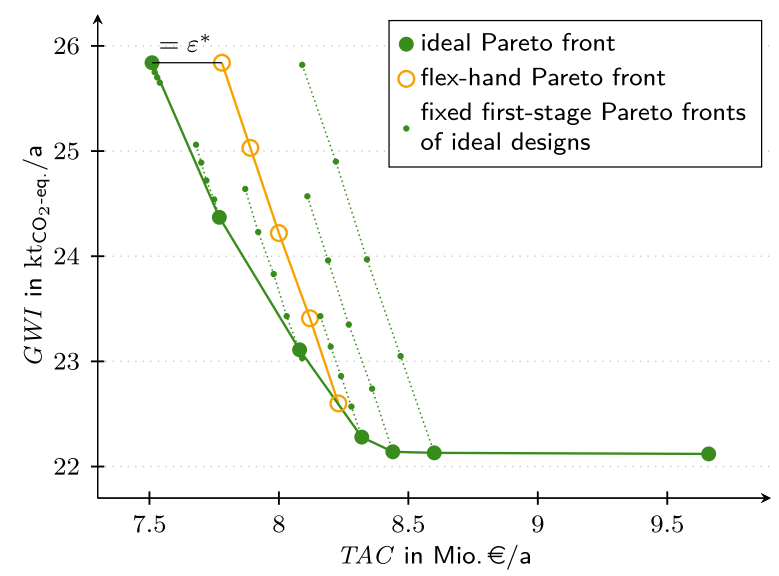

respectively. Hence, the maximal deviation for total annualized costs is $0.27 \mathrm{Mio} € € / \mathrm{a}$ which corresponds to a maximal loss of only $3.6 \%$ compared to the ideal design with minimized total annualized costs. Regarding the minimal global warming impact, the maximal deviation is only $2.17 \%$. Thus, the flexibility of the flex-hand design is very high regarding both objective functions.

When having a closer look at the identified design, we cannot identify a unique reason for its higher ability to adapt operation (Fig. 7).

In general, solutions with lower global warming impact prefer installing higher thermal power of combined heat and power engines, since the specific global warming impact of the electricity mix of the grid is higher than the impact of the combined heat and power engines in combination with absorption chillers. The flex-hand design does not show remarkable differences compared to the other ideal designs but provides an excellent compromise. Without the proposed approach, this highly flexible design would most likely not have been identified by the decision maker.

\subsection{The robust flex-hand design}

We now apply the robust flex-hand approach to the proposed case study taking uncertainties into account. The uncertainties are introduced in Sect. 3.1. Here, we consider three representative scenarios $\xi_{1}, \xi_{2}$, and $\xi_{3}$. Scenario $\xi_{2}$ corresponds to values of the problem without uncertainties discussed in Sect.3.2. In scenario $\xi_{1}$, we assume all uncertain values to take their smallest values within the uncertainty range and in scenario $\xi_{3}$ their largest values, respectively. However, any other scenario could be chosen.

To evaluate the robust flex-hand design, we compare the robust flex-hand Pareto fronts in all three scenarios to the flex-hand Pareto fronts generated for each scenario separately. Figure 8 shows that the flex-hand Pareto fronts generated for each scenario separately do not coincide with the robust flex-hand Pareto fronts. In scenario $\xi_{3}$, the robust flex-hand design leads to smaller total annualized costs than the flex-hand design computed for scenario $\xi_{3}$ but to a higher global warming impact. In total, the 


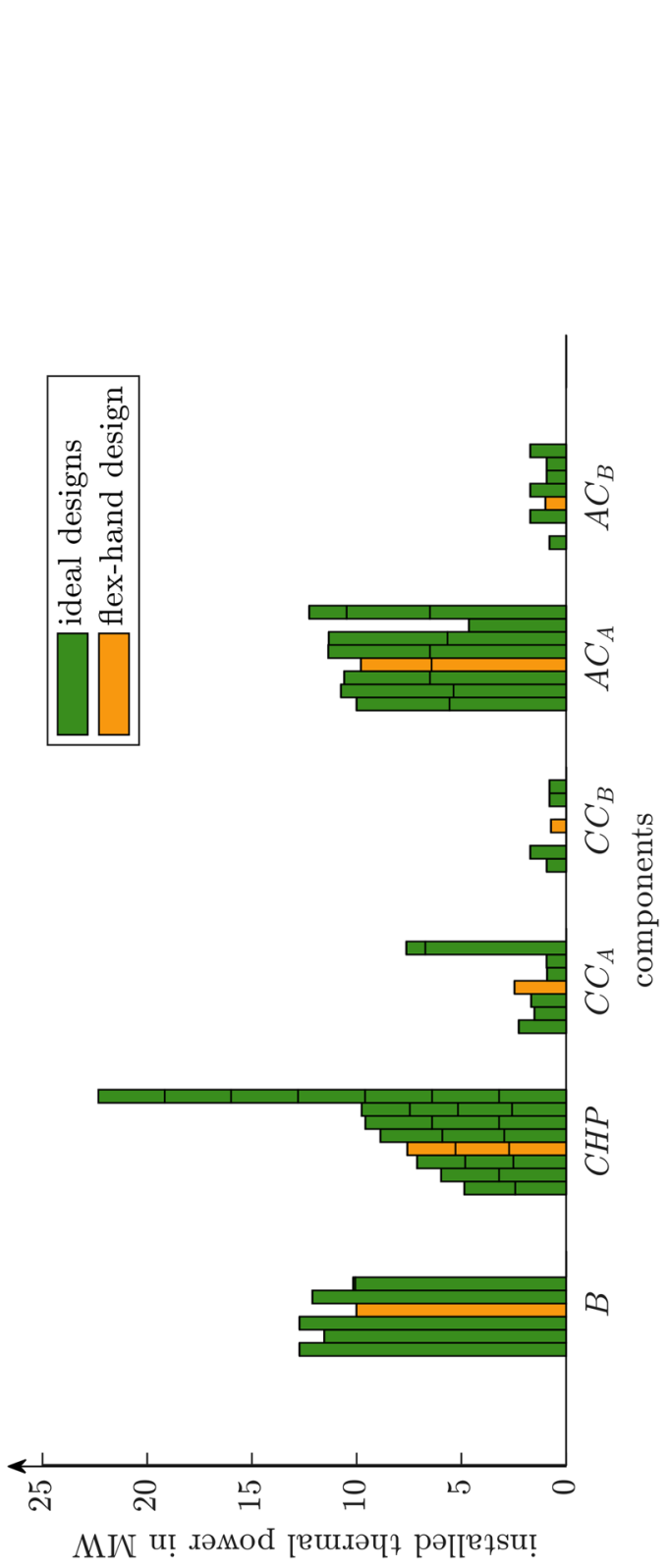

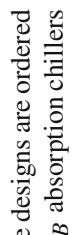

$\cong U^{\infty}$

芒官

氜

들

.

휼

؛

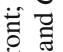

50

U

:

交

$\underset{0}{\stackrel{2}{2}}$

$\stackrel{2}{\Xi}$

대

.5

政

ปั

$\underset{0}{\stackrel{0}{*}}$

흏

$\Xi$

䒠

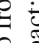

를 궁

ㅎ.

on

过

3

站

कo

프

宛見安

ธ。

要 00

娄灵

$\Xi \nsubseteq$

$\wedge$ 을

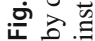




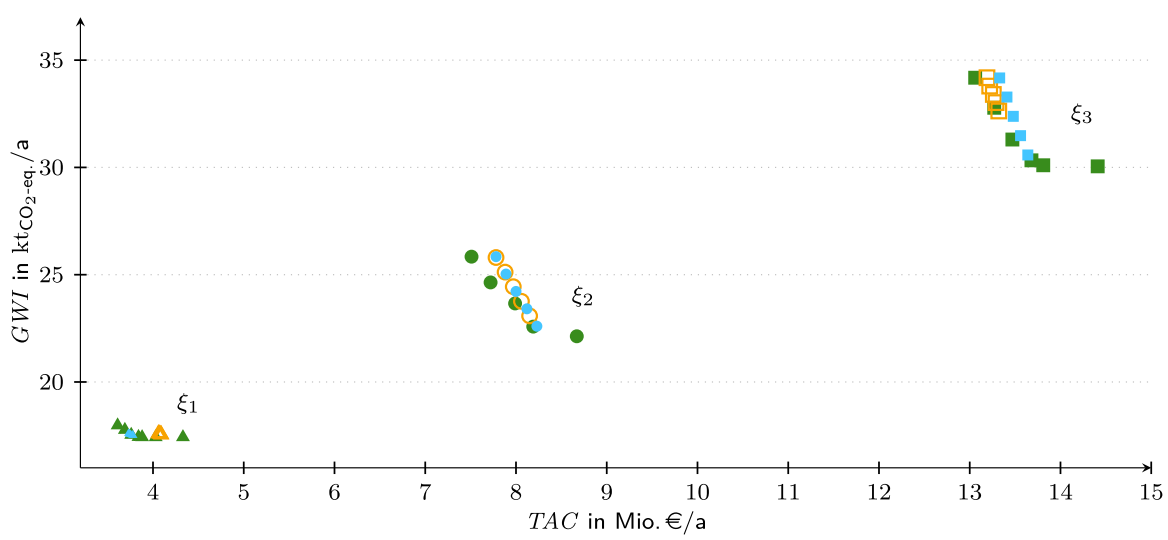

Fig. 8 Triangles, circles, and squares represent scenario $\xi_{1}, \xi_{2}$, and $\xi_{3}$, respectively; dark green filled marks: ideal Pareto front generated for each scenario separately, orange unfilled marks: robust flex-hand Pareto front in each scenario; small light blue marks: flex-hand Pareto front for separately considered scenarios; here, Pareto fronts are presented without normalization

Fig. 9 All Pareto fronts for scenario $\xi_{1}$; dark green filled triangles $(\mathbf{\Delta})$ : ideal Pareto front of scenario $\xi_{1}$; orange unfilled triangles $(\triangle)$ : robust flex-hand Pareto front in scenario $\xi_{1}$; small light blue triangles $(\Delta)$ : flex-hand Pareto front of scenario $\xi_{1}$; small light blue unfilled circles and squares (。 and $\square$ ): flex-hand Pareto front in scenario $\xi_{1}$ based on flex-hand design computed for scenario $\xi_{2}$ and $\xi_{3}$, respectively; here, Pareto fronts are presented without normalization

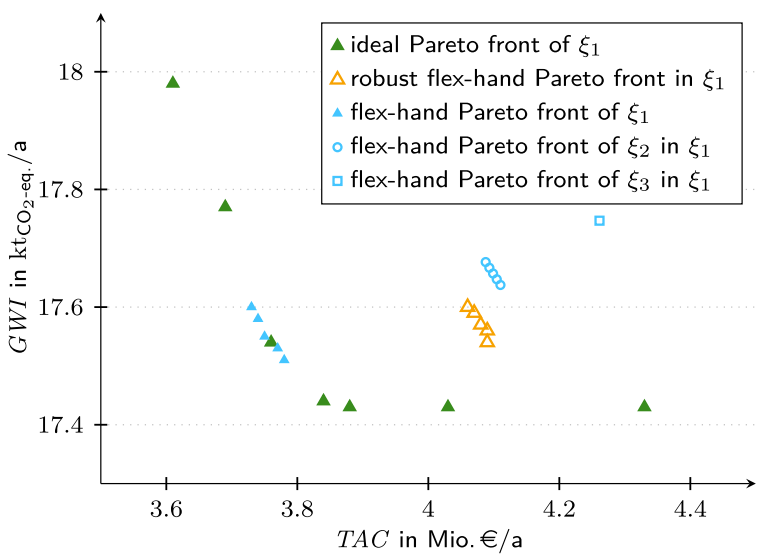

robust flex-hand Pareto front is less "streched out" than for the nominal case (Sect. 3.2) leading to an optimal distance $\bar{\varepsilon}^{*}$ of 0.625 . The reduced adaptability to the ideal Pareto fronts is due to the fact that the robust flex-hand Pareto fronts need to approximate three ideal Pareto fronts simultaneously, instead of just one Pareto front. Thus, a good performance of a flex-hand design in one scenario might lead to a poor performance in another scenario if uncertainties are not regarded during design (Fig. 9). In contrast, the robust flex-hand design is a compromise solution performing well in all three scenarios simultaneously.

In Fig. 9, we take a closer look on the computed Pareto fronts in scenario $\xi_{1}$. Here, the robust flex-hand design $(\Delta)$ clearly performs better than the flex-hand design identified for scenario $\xi_{2}(\circ)$ and the flex-hand design identified for scenario $\xi_{3}(\square)$. In scenario $\xi_{1}$, only the flex-hand Pareto front of scenario $\xi_{1}(\Delta)$ approximates the ideal Pareto front $(\Delta)$ better than the robust flex-hand Pareto front $(\Delta)$. However, the 


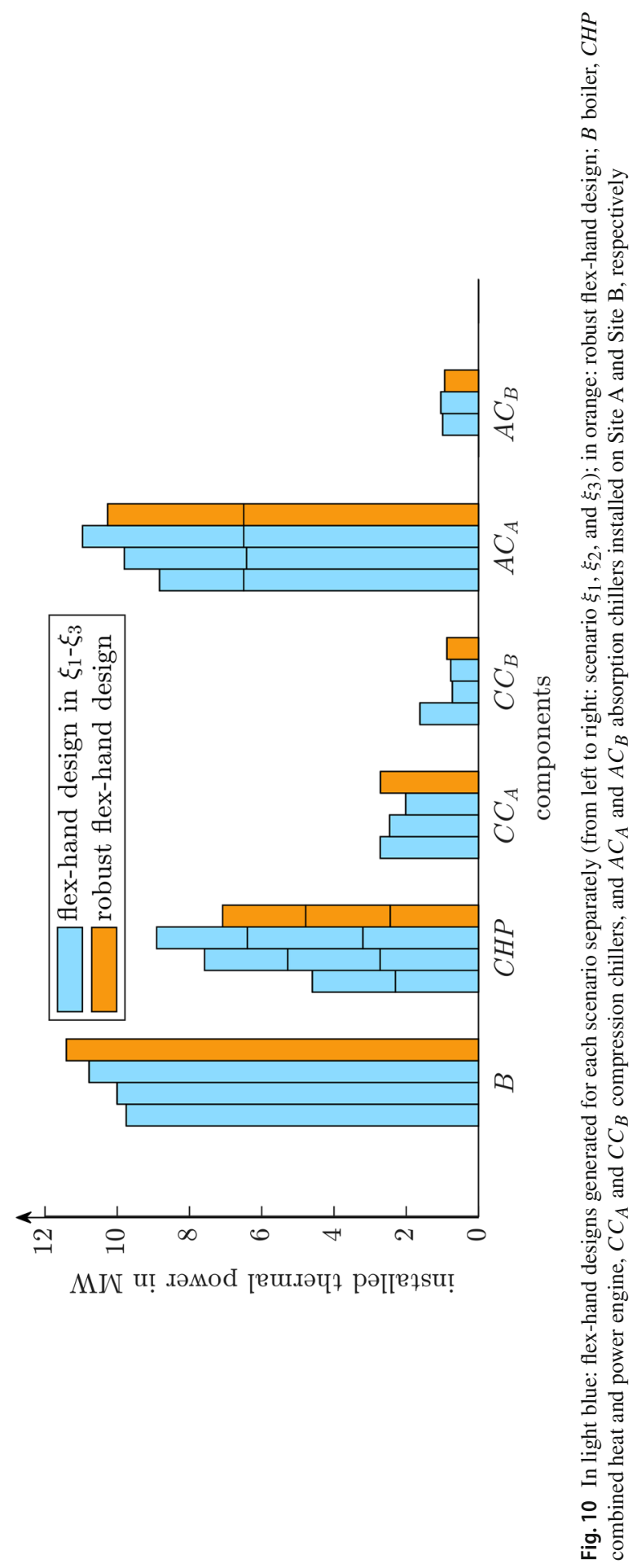


flex-hand design of scenario $\xi_{1}$ is infeasible for scenario $\xi_{2}$ and $\xi_{3}$. In contrast, the robust flex-hand design is feasible and performs well for all scenarios.

Having a closer look at the design (Fig. 10), we observe that the total thermal power of the three flex-hand designs increases from scenario $\xi_{1}$ to $\xi_{3}$. This is due to the fact that values of uncertain input parameter increase as well. With increasing demands and also increasing specific global warming impact of the electricity grid, larger combined heat and power engines and boilers are installed combined with a higher thermal power of absorption chillers and smaller compression chillers. The robust flex-hand design does not differ remarkably from the three flex-hand designs. Thus, the robust flex-hand approach is necessary to identify the excellent compromise given by the robust flex-hand design.

\section{Conclusions}

The sustainable optimization of energy systems is inherently a two-stage optimization problem with multiple decision criteria. Applying multi-objective optimization usually generates different designs for each point on the Pareto front. We propose the flex-hand approach for identifying a single design which performs well regarding all decision criteria. The idea of the flex-hand approach is to approximate the Pareto front with changing design options (ideal Pareto front) by a Pareto front with one fixed design for the whole front. The design leading to the minimal distance between both Pareto fronts is the design which is identified by the flex-hand approach. The identified design (flex-hand design) is able to adapt to all regarded criteria well, and thus, provides high flexibility to reach future aims for which focus might change between the considered objective functions.

Our real-world case study demonstrates the resulting high adaptability with respect to the considered criteria. For designing the sustainable energy system, we choose total annualized costs and the global warming impact as economical and environmental criteria, respectively. The calculated Pareto front of the flex-hand design is "stretched out" in comparison to the Pareto fronts obtained by operational optimization of designs lying on the ideal Pareto front. The objective function values of flex-hand design differ by less than $3.6 \%$ from the ideal values which highlights the excellent quality of the identified flex-hand design. The flex-hand design does not show remarkable differences compared to the designs lying on the ideal Pareto front. Thus, without the flex-hand approach, the decision maker would possibly not have chosen the identified design. This effect becomes even more pronounced when considering multiple scenarios simultaneously to account for uncertainty, in which case our approach is able to find a robust solution.

To conclude, the flex-hand approach takes advantage of the two-stage nature of energy systems to automatically select one single design which provides a high flexibility to adapt operation to all considered criteria.

Acknowledgements Open Access funding provided by Projekt DEAL. This work was supported by the Helmholtz Association under the Joint Initiative Energy System 2050-A Contribution of the Research Field Energy. 
Open Access This article is licensed under a Creative Commons Attribution 4.0 International License, which permits use, sharing, adaptation, distribution and reproduction in any medium or format, as long as you give appropriate credit to the original author(s) and the source, provide a link to the Creative Commons licence, and indicate if changes were made. The images or other third party material in this article are included in the article's Creative Commons licence, unless indicated otherwise in a credit line to the material. If material is not included in the article's Creative Commons licence and your intended use is not permitted by statutory regulation or exceeds the permitted use, you will need to obtain permission directly from the copyright holder. To view a copy of this licence, visit http://creativecommons.org/licenses/by/4.0/.

\section{Appendix A: Model formulation}

In the following, we provide the problem formulation of the robust flex-hand optimization for the distributed energy supply system considered in our case study based on the model formulation provided by Voll et al. (2013) (Sect.3). In the case study, we consider the total annualized costs $T A C$ and the global warming impact GWI as objective functions. Uncertainties are regarded for tariffs for purchasing gas $\widetilde{p}^{\text {gas }}(\xi)$ and electricity $\widetilde{p}^{\text {el,buy }}(\xi)$ as well as for selling electricity $\widetilde{p}^{\text {el,sell }}(\xi)$. Furthermore, the specific global warming impact of the electricity mix of the grid $\widetilde{G W I}^{e l}(\xi)$ is assumed to be uncertain as well. In the constraints, the energy balances are affected by uncertain energy demands $\widetilde{\mathscr{E}}^{\text {heat }}(\xi), \widetilde{\tilde{E}}^{\text {cool }}(\xi)$, and $\widetilde{\dot{E}}^{e l}(\xi)$.

$$
\begin{aligned}
& \min \bar{\varepsilon} \\
& \text { s. t. } \overline{T A C}\left(\dot{U}, \dot{U}^{e l, b u y}, \dot{V}^{e l, s e l l}, \gamma, \dot{V}^{N} ; \xi, j\right)-\overline{\operatorname{TAC}}^{*}(\xi, j) \leq \bar{\varepsilon} \\
& \forall \xi \in \mathcal{U}, j \in[N(\xi)] \\
& \overline{G W I}\left(\dot{U}, \dot{U}^{e l, b u y}, \dot{V}^{e l, s e l l} ; \xi, j\right)-\overline{G W I}^{*}(\xi, j) \leq \bar{\varepsilon} \\
& \forall \xi \in \mathcal{U}, j \in[N(\xi)] \\
& \sum_{k \in B \cup C H P} \dot{V}_{k t}(\xi, j)-\sum_{k \in A C} \dot{U}_{k t}(\xi, j)=\widetilde{\tilde{E}}_{t}^{\text {heat }}(\xi) \\
& \forall t \in[T], \xi \in \mathcal{U}, j \in[N(\xi)] \\
& \sum_{k \in A C \cup C C} \dot{V}_{k t}(\xi, j)=\widetilde{E}_{t}^{\text {cool }}(\xi) \\
& \forall t \in[T], \xi \in \mathcal{U}, j \in[N(\xi)] \\
& \sum_{k \in C H P} \dot{V}_{k t}^{e l}(\xi, j)-\sum_{k \in C C} \dot{U}_{k t}(\xi, j) \\
& +\dot{U}_{t}^{e l, b u y}(\xi, j)-\dot{V}_{t}^{e l, s e l l}(\xi, j)=\widetilde{E}_{t}^{e l}(\xi) \\
& \forall t \in[T], \xi \in \mathcal{U}, j \in[N(\xi)] \\
& \sum_{h \in[H]} \gamma_{k h} \leq 1 \\
& \forall k \in \mathcal{K} \\
& \gamma_{k h} \cdot \dot{V}_{k h}^{N, l b} \leq \dot{V}_{k h}^{N} \leq \gamma_{k h} \cdot \dot{V}_{k h+1}^{N, l b} \\
& \forall k \in \mathcal{K}, \forall h \in[H] \\
& \rho^{\min } \cdot \sum_{h \in[H]} \dot{V}_{k h}^{N} \leq \dot{V}_{k t}(\xi, j) \leq \sum_{h \in[H]} \dot{V}_{k h}^{N} \quad \forall k \in \mathcal{K}, t \in[T], \xi \in \mathcal{U}, j \in[N(\xi)] \\
& \dot{V}_{k t}(\xi, j)=\eta_{k} \cdot \dot{U}_{k t}(\xi, j) \\
& \forall k \in \mathcal{K}, t \in[T], \xi \in \mathcal{U}, j \in[N(\xi)] \\
& \dot{V}_{k t}^{e l}(\xi, j)=\eta_{k}^{t o t} \cdot \dot{U}_{k t}(\xi, j)-\dot{V}_{k t}(\xi, j) \\
& \forall k \in C H P, t \in[T], \xi \in \mathcal{U}, j \in[N(\xi)] \\
& \bar{\varepsilon} \in \mathbb{R}_{+} \\
& \dot{U}^{\text {el, buy }}(\xi, j), \dot{V}^{\text {el,sell }}(\xi, j), \dot{V}^{e l}(\xi, j), \dot{U}(\xi, j), \dot{V}(\xi, j) \in \mathbb{R}_{+}^{|\mathcal{K}| \times T} \quad \forall \xi \in \mathcal{U}, j \in[N(\xi)] \\
& \gamma \in\{0,1\}^{|\mathcal{K}| \times H}, \dot{V}^{N} \in \mathbb{R}_{+}^{|\mathcal{K}| \times H}
\end{aligned}
$$


The total annualized costs $T A C$ and the global warming impact $G W I$ are defined by

$$
\begin{aligned}
\text { TAC } & \left(\dot{U}, \dot{U}^{\text {el,buy }}, \dot{V}^{e l, s e l l}, \gamma, \dot{V}^{N} ; \xi, j\right) \\
= & \sum_{t \in[T]}\left[\Delta \tau _ { t } \left(\tilde{p}^{\text {gas }}(\xi) \cdot \sum_{k \in B \cup C H P} \dot{U}_{k t}(\xi, j)+\tilde{p}^{\text {el,buy }}(\xi) \cdot \dot{U}_{t}^{e l, b u y}(\xi, j)\right.\right. \\
& \left.\left.-\tilde{p}^{\text {el,sell }}(\xi) \cdot \dot{V}_{t}^{e l, s e l l}(\xi, j)\right)\right] \\
& +\sum_{k \in \mathcal{K}}\left(\frac{1}{P V F}+p_{k}^{m}\right) \cdot \underbrace{\sum_{h \in[H]}\left[\gamma_{k h} \cdot \kappa_{k h}+m_{k h} \cdot\left(\dot{V}_{k h}^{N}-\gamma_{k h} \dot{V}_{k h}^{N, l b}\right)\right]}_{=: I N V E S T_{k}} \\
G W I & \left(\dot{U}, \dot{U}^{e l, b u y}, \dot{V}^{e l, s e l l} ; \xi, j\right) \\
= & \sum_{t \in[T]} \Delta \tau_{t} \sum_{k \in B \cup C H P} \dot{U}_{k t}(\xi, j) \cdot G W I^{\text {gas }} \\
& \left.+\left(\dot{U}_{t}^{e l, b u y}(\xi, j)-\dot{V}_{t}^{e l, s e l l}(\xi, j)\right) \cdot \widetilde{G W I}^{e l}(\xi)\right] .
\end{aligned}
$$

Bars above the total annualized costs TAC and the global warming impact GWI in the optimization problem denote the normalization of the objective values. Objective values on the normalized ideal Pareto fronts are denoted by $\left(\overline{T A C}^{*}(\xi, j), \overline{G W I}^{*}(\xi, j)\right)$ for each point $j \in[N(\xi)]$ and each scenario $\xi$ in the uncertainty set $\mathcal{U}$.

The duration of a time step $t \in[T]$ is given by $\Delta \tau_{t}$. Maintenance costs are determined by the share $p_{k}^{m}$ of the investment costs INVEST $T_{k}$. The investment costs $I N V E S T_{k}$ are annualized by the present value factor $P V F . G W I^{\text {gas }}$ represent the specific global warming impact of purchased gas. Purchased and sold electricity is denoted by $\dot{U}_{t}^{e l, b u y}$ and $\dot{V}_{t}^{e l, s e l l}$, respectively. $\dot{U}_{k t}$ and $\dot{V}_{k t}$ specifies input and output energy flows in time step $t$ of component $k \in \mathcal{K}$. Components include boilers $B$, combined heat and power engines $C H P$, absorption chillers $A C$, and compression chillers $C C$. Input and output energy flows are coupled by the thermal efficiency $\eta_{k}$. For combined heat and power engines, the total efficiency $\eta_{k}^{\text {tot }}$ is given by the sum of the thermal and the electrical efficiency $\eta_{k}^{\text {tot }}=\eta_{k}+\eta_{k}^{e l}$. The minimal part-load of a component $k$ is defined by the fraction $\rho^{\mathrm{min}}$ of the installed nominal capacity. The operational variables $\dot{U}_{k t}, \dot{V}_{k t}, \dot{U}_{t}^{e l, b u y}$ and $\dot{V}_{t}^{\text {el, sell }}$ depending on time step $t$ relate to the second-stage variables $x^{s}$.

The first constraints (7) and (8) refer to the additional constraints to limit the binary $\varepsilon$-indicator. The energy balances for heating, cooling and electricity are given in Eqs. (9)-(11). Constraint (12) is part of the linearization of the investment costs $I_{N V E S T}$ which is explained in detail in the following paragraph. The nominal installed thermal power and the operational power are limited by constraints (13) and (14), respectively. Equations (15) and (16) relate output energy to the input energy depending on the efficiency. 
Fig. 11 Piecewise linearization of the investment costs INVEST $T_{k}$ of a newly installed component $k$ is presented. Here, $h$ is the active line segment; thus, the binary variable $\gamma_{k h}$ is equal to 1

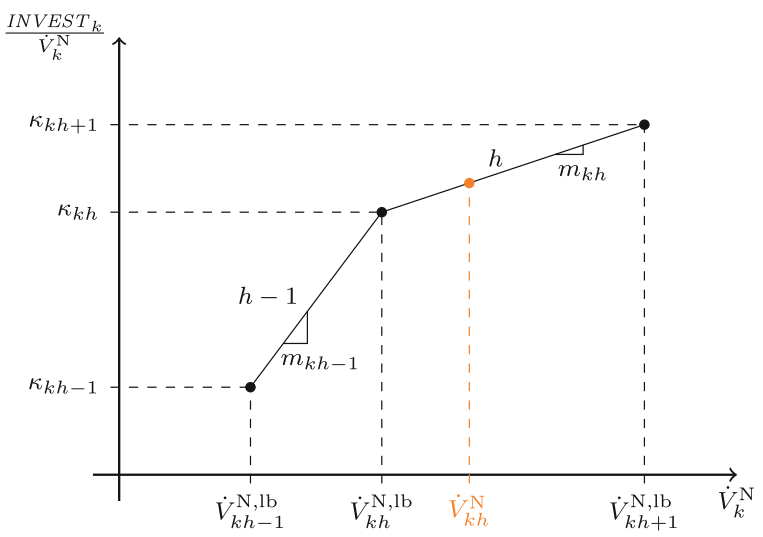

The investment costs INVEST $k$ of a newly installed component $k$ are linearized by piecewise linearization with $\sum_{h \in[H]}\left[\gamma_{k h} \cdot \kappa_{k h}+m_{k h} \cdot\left(\dot{V}_{k h}^{N}-\gamma_{k h} \dot{V}_{k h}^{N, l b}\right)\right]$ (see Fig. 11). $m_{k h}$ is the slope for each line segment $h \in[H]$ and is defined by

$$
m_{k h}:=\frac{\kappa_{k h+1}-\kappa_{k h}}{\dot{V}_{k h+1}^{N, l b}-\dot{V}_{k h}^{N, l b}} \quad \forall k \in \mathcal{K}, h \in[H] .
$$

Here, parameters $\dot{V}_{k h+1}^{N, l b}$ and $\dot{V}_{k h}^{N, l b}$ represent the nominal capacities of the lower and upper supporting point of line segment $h$ and parameters $\kappa_{k h}$ and $\kappa_{k h+1}$ the corresponding specific investment costs. Binary variables $\gamma_{k h}$ determine if line segment $h$ is active $\left(\gamma_{k h}=1\right)$. Since the sum $\sum_{h \in[H]} \gamma_{k h}$ is equal to or less than 1 (Eq. (12)), only one line segment can be active at the time. Thus, only one value for the nominal capacity $\dot{V}_{k h}^{N}$ of all line segments is unequal to 0 ; hence, the nominal capacity $\dot{V}_{k}^{N}$ of an installed component $k$ is given by the sum $\sum_{h \in[H]} \dot{V}_{k h}^{N}$. In the case study, first-stage variables $x^{f}$ are the design decisions $\dot{V}_{k h}^{N}$ and $\gamma_{k h}$ with investment costs $I N V E S T_{k}$ for each installed component $k$.

\section{References}

Abubaker A, Baharum A, Alrefaei M (2014) Good solution for multi-objective optimization problem. AIP Conf Proc 1605(1):1147-1152. https://doi.org/10.1063/1.4887752

Aissi H, Bazgan C, Vanderpooten D (2009) Min-max and min-max regret versions of combinatorial optimization problems: a survey. Eur J Oper Res 197(2):427-438. https://doi.org/10.1016/j.ejor.2008. 09.012

Antipova E, Pozo C, Guillén-Gosálbez G, Boer D, Cabeza L, Jiménez L (2015) On the use of filters to facilitate the post-optimal analysis of the Pareto solutions in multi-objective optimization. Comput Chem Eng 74:48-58. https://doi.org/10.1016/j.compchemeng.2014.12.012

Baumann H, Tillman AM (2004) The Hitch Hiker's Guide to LCA. Studentlitteratur AB

Ben-Tal A, Goryashko A, Guslitzer E, Nemirovski A (2004) Adjustable robust solutions of uncertain linear programs. Math Prog 99(2):351-376. https://doi.org/10.1007/s10107-003-0454-y

Beume N, Fonseca CM, Lopez-Ibanez M, Paquete L, Vahrenhold J (2009) On the complexity of computing the hypervolume indicator. IEEE Trans Evol Comput 13(5):1075-1082. https://doi.org/10.1109/ TEVC.2009.2015575 
Branke J, Deb K, Dierolf H, Osswald M (2004) Finding knees in multi-objective optimization. Lecture notes in computer science. Springer, Berlin, pp 722-731. https://doi.org/10.1007/978-3-540-302179_73

Broverman SA (2010) Mathematics of investment and credit, 5th edn. ACTEX Publications, Inc, New Hartford

Carvalho M, Lozano MA, Serra LM (2012) Multicriteria synthesis of trigeneration systems considering economic and environmental aspects. Appl Energy 91(1):245-254. https://doi.org/10.1016/j.apenergy. 2011.09.029

Chen SJ, Hwang CL (1992) Fuzzy multiple attribute decision making methods. Springer, Berlin, pp 289486. https://doi.org/10.1007/978-3-642-46768-4_5

Das I (1999) A preference ordering among various Pareto optimal alternatives. Struct Optim 18(1):30-35. https://doi.org/10.1007/BF01210689

Das I, Dennis JE (1998) Normal-boundary intersection: a new method for generating the Pareto surface in nonlinear multicriteria optimization problems. SIAM J Optim 8(3):631-657. https://doi.org/10.1137/ S1052623496307510

de la Fuente D, Vega-Rodríguez MA, Pérez CJ (2018) Automatic selection of a single solution from the Pareto front to identify key players in social networks. Knowl-Based Syst 160:228-236. https://doi. org/10.1016/j.knosys.2018.07.018

Duckstein L, Opricovic S (1980) Multiobjective optimization in river basin development. Water Resour Res 16(1):14-20. https://doi.org/10.1029/WR016i001p00014

Ehrgott M (2005) Multicriteria optimization, 2nd edn. Springer, Berlin

Eskelinen P, Miettinen K, Klamroth K, Hakanen J (2008) Pareto navigator for interactive nonlinear multiobjective optimization. OR Spectr 32(1):211-227. https://doi.org/10.1007/s00291-008-0151-6

Gabrel V, Murat C, Thiele A (2014) Recent advances in robust optimization: an overview. Eur J Oper Res 235(3):471-483

Gabrielli P, Fürer F, Mavromatidis G, Mazzotti M (2019) Robust and optimal design of multi-energy systems with seasonal storage through uncertainty analysis. Appl Energy 238:1192-1210. https://doi.org/10. 1016/j.apenergy.2019.01.064

Guillén-Gosálbez G (2011) A novel MILP-based objective reduction method for multi-objective optimization: application to environmental problems. Comput Chem Eng 35(8):1469-1477. https://doi.org/10. 1016/j.compchemeng.2011.02.001

Guo L, Liu W, Cai J, Hong B, Wang C (2013) A two-stage optimal planning and design method for combined cooling, heat and power microgrid system. Energy Convers Manag 74:433-445. https://doi.org/10. 1016/j.enconman.2013.06.051

Hennen M, Postels S, Voll P, Lampe M, Bardow A (2017) Multi-objective synthesis of energy systems: efficient identification of design trade-offs. Comput Chem Eng 97:283-293. https://doi.org/10.1016/ j.compchemeng.2016.10.010

Hwang CL, Yoon K (1981) Methods for multiple attribute decision making. Springer, Berlin, pp 58-191. https://doi.org/10.1007/978-3-642-48318-9_3

IBM Corporation (2015) IBM ILOG CPLEX Optimization Studio, Version 12.6. User Guide

Ide J, Schöbel A (2016) Robustness for uncertain multi-objective optimization: a survey and analysis of different concepts. OR Spectr 38(1):235-271. https://doi.org/10.1007/s00291-015-0418-7

Jing R, Wang M, Zhang Z, Liu J, Liang H, Meng C, Shah N, Li N, Zhao Y (2019) Comparative study of posteriori decision-making methods when designing building integrated energy systems with multiobjectives. Energ Build 194:123-139. https://doi.org/10.1016/j.enbuild.2019.04.023

Kasperski A, Zieliński P (2017) Robust recoverable and two-stage selection problems. Discrete Appl Math 233:52-64. https://doi.org/10.1016/j.dam.2017.08.014

Lemos LP, Lima EL, Pinto JC (2018) New decision making criterion for multiobjective optimization problems. Ind Eng Chem Res 57(3):1014-1025. https://doi.org/10.1021/acs.iecr.7b04196

Li Z, Liao H, Coit DW (2009) A two-stage approach for multi-objective decision making with applications to system reliability optimization. Reliab Eng Syst Safe 94(10):1585-1592. https://doi.org/10.1016/ j.ress.2009.02.022

Lin F, Leyffer S, Munson T (2016) A two-level approach to large mixed-integer programs with application to cogeneration in energy-efficient buildings. Comput Optim Appl 65(1):1-46. https://doi.org/10.1007/ s10589-016-9842-0 
Majewski DE, Wirtz M, Lampe M, Bardow A (2017) Robust multi-objective optimization for sustainable design of distributed energy supply systems. Comput Chem Eng 102:26-39. https://doi.org/10.1016/ j.compchemeng.2016.11.038

Mattson CA, Messac A (2003) Concept selection using s-Pareto frontiers. AIAA J 10(2514/2):2063

McCarl BA, Rosenthal RE (2016) McCarl GAMS user guide. Version 24:7

Miettinen K (2008) Introduction to multiobjective optimization: noninteractive approaches. Springer, Berlin, pp 1-26. https://doi.org/10.1007/978-3-540-88908-3_1

Miettinen K, Mäkelä MM (1996) NIMBUS — interactive method for nondifferentiable multiobjective optimization problems. In: Multi-objective programming and goal programming. Springer, Berlin, pp 50-57. https://doi.org/10.1007/978-3-642-87561-8_5

Miettinen K, Mäkelä MM (2002) On scalarizing functions in multiobjective optimization. OR Spectr 24(2):193-213. https://doi.org/10.1007/s00291-001-0092-9

Mota B, Gomes MI, Carvalho A, Barbosa-Povoa AP (2015) Towards supply chain sustainability: economic, environmental and social design and planning. J Clean Prod 105:14-27. https://doi.org/10.1016/j. jclepro.2014.07.052

Opricovic S, Tzeng GH (2004) Compromise solution by MCDM methods: a comparative analysis of VIKOR and TOPSIS. Eur J Oper Res 156(2):445-455. https://doi.org/10.1016/S0377-2217(03)00020-1

Padhye N, Deb K (2011) Multi-objective optimisation and multi-criteria decision making for FDM using evolutionary approaches. Springer, London, p 219. https://doi.org/10.1007/978-0-85729-652-8_7

Poursoltani M, Delage E (2019) Adjustable robust optimization reformulations of two-stage worst-case regret minimization problems. Tech. rep., Optimization Online

Pérez CJ, Vega-Rodríguez MA, Reder K, Flörke M (2017) A multi-objective artificial bee colony-based optimization approach to design water quality monitoring networks in river basins. J Clean Prod 166:579-589. https://doi.org/10.1016/j.jclepro.2017.08.060

Quintana D, Denysiuk R, Garcia-Rodriguez S, Gaspar-Cunha A (2017) Portfolio implementation risk management using evolutionary multiobjective optimization. Appl Sci 7(10):1079. https://doi.org/ 10.3390/app7101079

Rachmawati L, Srinivasan D (2009) Multiobjective evolutionary algorithm with controllable focus on the knees of the Pareto front. IEEE Trans Evol Comput 13(4):810-824. https://doi.org/10.1109/TEVC. 2009.2017515

Shang Z, Kokossis A (2005) A systematic approach to the synthesis and design of flexible site utility systems. Chem Eng Sci 60(16):4431-4451. https://doi.org/10.1016/j.ces.2005.03.015

Soyster AL (1973) Technical note-convex programming with set-inclusive constraints and applications to inexact linear programming. Oper Res 21(5):1154-1157. https://doi.org/10.1287/opre.21.5.1154

Srinivasan V, Shocker AD (1973) Linear programming techniques for multidimensional analysis of preferences. Psychometrika 38(3):337-369. https://doi.org/10.1007/BF02291658

Sun G, Zhang H, Fang J, Li G, Li Q (2018) A new multi-objective discrete robust optimization algorithm for engineering design. Appl Math Model 53:602-621. https://doi.org/10.1016/j.apm.2017.08.016

Taboada HA, Baheranwala F, Coit DW, Wattanapongsakorn N (2007) Practical solutions for multi-objective optimization: an application to system reliability design problems. Reliab Eng Syst Safe 92(3):314322. https://doi.org/10.1016/j.ress.2006.04.014

Tock L, Maréchal F (2015) Decision support for ranking Pareto optimal process designs under uncertain market conditions. Comput Chem Eng 83:165-175. https://doi.org/10.1016/j.compchemeng.2015.06. 009

Vallerio M, Hufkens J, Van Impe J, Logist F (2015) An interactive decision-support system for multi-objective optimization of nonlinear dynamic processes with uncertainty. Expert Syst Appl 42(21):7710-7731. https://doi.org/10.1016/j.eswa.2015.05.038

Voll P, Klaffke C, Hennen M, Bardow A (2013) Automated superstructure-based synthesis and optimization of distributed energy supply systems. Energy 50:374-388. https://doi.org/10.1016/j.energy.2012.10. 045

Wang Y, Wang Y, Huang Y, Li F, Zeng M, Li J, Wang X, Zhang F (2019) Planning and operation method of the regional integrated energy system considering economy and environment. Energy 171:731-750. https://doi.org/10.1016/j.energy.2019.01.036

Wierzbicki AP (2007) Reference point approaches and objective ranking. In: Branke J, Deb K, Miettinen K, Slowinski R (eds) Practical approaches to multi-objective optimization. Schloss Dagstuhl LeibnizZentrum für Informatik, Dagstuhl, no. 06501 in Dagstuhl seminar proceedings 
Yanıkoğlu I, Gorissen BL, den Hertog D (2019) A survey of adjustable robust optimization. Eur J Oper Res 277(3):799-813

Zelany M (1974) A concept of compromise solutions and the method of the displaced ideal. Comput Oper Res 1(3-4):479-496. https://doi.org/10.1016/0305-0548(74)90064-1

Zio E, Bazzo R (2011) A clustering procedure for reducing the number of representative solutions in the Pareto front of multiobjective optimization problems. Eur J Oper Res 210(3):624-634. https://doi.org/ 10.1016/j.ejor.2010.10.021

Zitzler E, Thiele L, Laumanns M, Fonseca CM, da Fonseca VG (2003) Performance assessment of multiobjective optimizers: an analysis and review. IEEE Trans Evol Comput 7(2):117-132. https://doi.org/ 10.1109/TEVC.2003.810758

Publisher's Note Springer Nature remains neutral with regard to jurisdictional claims in published maps and institutional affiliations.

\section{Affiliations}

\section{Dinah Elena Hollermann ${ }^{1}$. Marc Goerigk ${ }^{2}$. Dörthe Franzisca Hoffrogge ${ }^{1}$. Maike Hennen ${ }^{1}$. André Bardow ${ }^{1,3}$}

Dinah Elena Hollermann

dinah.hollermann@rwth-aachen.de

Dörthe Franzisca Hoffrogge

doerthe.hoffrogge@rwth-aachen.de

Maike Hennen

maike.hennen@rwth-aachen.de

André Bardow

andre.bardow@1tt.rwth-aachen.de

1 Institute of Technical Thermodynamics, RWTH Aachen University, 52056 Aachen, Germany

2 Network and Data Science Management, University of Siegen, 57072 Siegen, Germany

3 Institute of Energy and Climate Research, Energy Systems Engineering (IEK-10), Forschungszentrum Jülich GmbH, 52425 Jülich, Germany 